\title{
Split control plane functionality in millimeter-wave overlay access
}

\author{
Richard J. Weiler and Wilhelm Keusgen \\ Fraunhofer Heinrich Hertz Institute \\ Berlin, Germany \\ \{richard.weiler, wilhelm.keusgen\}@ @hi.fraunhofer.de
}

\author{
Ilario Filippini and Antonio Capone \\ Dipartimento di Elettronica, Informazione e Bioingegneria \\ Politecnico di Milano \\ Milano, Italy \\ \{ilario.filippini, antonio.capone\}@ polimi.it
}

\begin{abstract}
The millimeter-wave band is a candidate to overcome the spectrum shortage in future mobile radio networks. As the coverage area of base stations operating at these frequencies is expected to be more limited than at the sub $6 \mathrm{GHz}$ bands, they will supplement existing base stations instead of replacing them. With these additional small cells a split plane architecture becomes an interesting architectural candidate, where capacity is provided by the small cells while continuous connectivity is guaranteed as it is available today. The introduction of such a split between the control and the data plane introduces new options and degrees of freedom of system and protocol design that are investigated in this paper.
\end{abstract}

Index Terms-heterogeneous networks, millimeter-wave communication, $60 \mathrm{GHz}, 5 \mathrm{G}$, HetNet, small cell

\section{INTRODUCTION}

Network densification and spectrum extension are two trends in the development of mobile radio networks [1], [2]. Adding small cell base stations in areas of existing macro cellular coverage promises to increase the network capacity as well as scalability and is a form of densification. This is commonly known as heterogeneous networks (HetNet). In current deployments the small cells often use the same frequency band as the macro cell, causing increasing levels of interference. Leveraging previously unused frequency resources in the millimeter-wave bands is a form of spectrum extension and promises decreased interference as well as increased capacity.

As we will discuss in section III the radio channel at millimeter-wave frequencies is much more challenging than the legacy ones in the sub $6 \mathrm{GHz}$ range. In order to maintain seamless connectivity in these bands recently a split plane approach was proposed where the control plane remains on the legacy frequency bands and the data (or user) plane can be moved to the millimeter-wave bands [3].

The millimeter-wave frequency band, ranging from $30 \mathrm{GHz}$ to $300 \mathrm{GHz}$, offers much wider bandwidths of contiguous spectrum than the legacy bands. During the last years a number of communication standards, using the widely license free available $60 \mathrm{GHz}$ band, have been defined. These standards however target indoor WPAN applications and are not designed for outdoor environments.

Adding an overlay of millimeter-wave capable small cells to the HetNet leads to additional control and user planes.
This gives new options for the logical localization of network functionality on the planes.

In this paper we investigate the special requirements for the control plane with respect to millimeter-wave specific characteristics and we discuss the resulting options for the additional planes. We analyze which control functionality must be on the millimeter-wave link, which can be chosen arbitrarily and which is bound to the macro cellular control plane. For that purpose the existing $60 \mathrm{GHz}$ indoor communication standards are used as a starting point.

Section II introduces the small cell overlay system architecture in more detail. The existing indoor communication standards are studied in section IV. Section V investigates the options for the split plane concept.

\section{SyStem ARCHITECTURE}

The millimeter-wave overlay system architecture with split user and control planes is shown in Figure 1. A macro base station (BS) provides coverage to all mobile terminals (UE) within its coverage area. Millimeter-wave small cell base stations are placed within the area of the macro base station and provide a smaller area of coverage with a millimeter-wave access link. The small cell base stations are connected to the macro base station via a high capacity backhaul. They can be in an active or inactive state, depending on the presence of users within their coverage area.

The user terminal possesses two wireless interfaces, one for the legacy connection to the macro cell (e.g. LTE) and one for the connection to a millimeter-wave link. In general the UE is connected to the small cell via the millimeter-wave link when necessary. At the same time the UE is attached to the macro $\mathrm{BS}$ via the legacy communication link.

On each physical connection control and/or user data can be transported. Thus, when the UE is connected on both the legacy and the millimeter-wave interface, two control and two data connections exist. One of each to the macro cell and one to the small cell. In the rest of this paper the first one will be referred to as macro cell or legacy connection and the second one as small cell or millimeter-wave connection.

\section{MILLIMETER-WAVE SPECIFICS}

The millimeter-wave band between 30 and $300 \mathrm{GHz}$ recently received increasing interest for new communication 


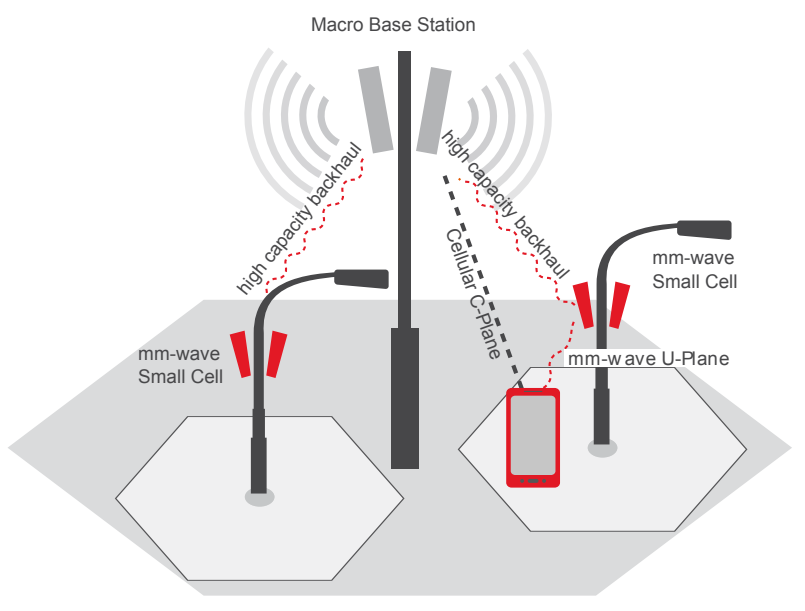

Figure 1. System architecture

standards to overcome the shortage of spectrum [1]. The increase in carrier frequency however leads to a higher pathloss. In free-space conditions the Friis equation shows that the received signal power is inversely proportional to the carrier frequency.

Several measurement campaigns of outdoor millimeterwave channels were reported recently [4]-[7]. The time dynamic behavior of the link and especially of the spatial multipath components is important for the design and parametrization of the beam forming algorithms in the millimeter-wave band. An analysis of channel measurements performed at 60 $\mathrm{GHz}$ in a busy urban outdoor access scenario shows that the different multipath components arriving at the receiver can be grouped into different classes, according to their availability over time [8]. Figure 2 shows the behavior of the omnidirectional channel impulse response for static transmitter and receiver locations on a busy urban street over a time of 50 seconds (see [6]-[8] for more details). Multipath components with a path loss of less than $120 \mathrm{~dB}$ are shown in blue. This threshold represents the maximum allowable path loss for communication and depends on the system implementation (e.g. antenna gain, noise figure, transmit power, modulation). It was chosen arbitrarily here. The distance between transmitter and receiver was 25 meters, which can be clearly seen by the constant path of the unblocked line of sight component at around $80 \mathrm{~ns}$ delay. Other multipath components at higher delays are caused by reflecting objects, such as the ground, surrounding buildings, street furniture, cars, etc. It can be seen that some exist throughout the measurement and are only interrupted while others only appear for a short period of time. In order to counter the higher path loss compared to sub $6 \mathrm{GHz}$ communication systems, millimeter-wave communication systems have to use directional antennas with high gain. To support mobile devices in outdoor environments the direction of the beam will need to adapt to the changing radio channel very fast, thus making electronic beam forming or beam steering, such as beam forming arrays necessary.

A thorough statistical analysis of this multipath compo-

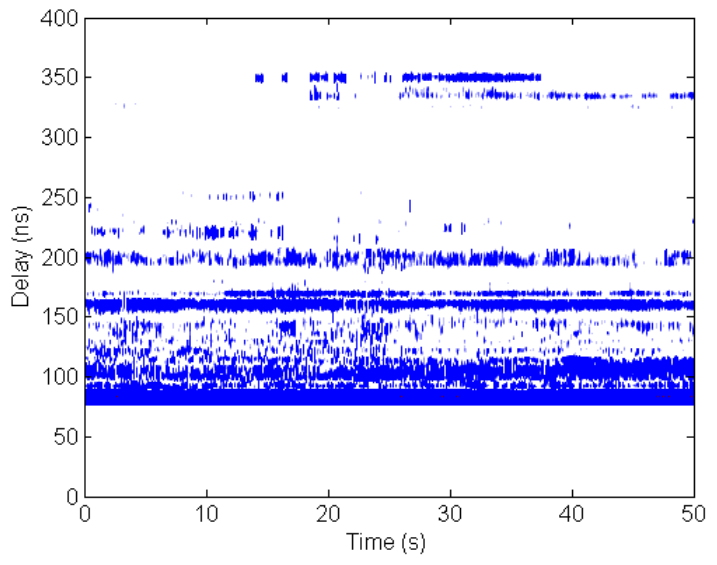

Figure 2. Time evolution of $60 \mathrm{GHz}$ outdoor channel impulse response

nent behavior over time is still open work. When assuming a blocked line of sight however, there are still a number of candidate multipath components that could be used to establish a directional link between the transmitter and the receiver, assuming that different multipath components stem from different spatial directions. The frequent interruption of some multipath components however indicates that a frequent switching between spatial directions might be necessary for a stable connection.

In cases when all multipath components between the transmitter and the receiver are to weak to support a communication link, the connection would have to fall back to the legacy connection.

\section{EXISTING $60 \mathrm{GHZ}$ INDOOR COMMUNICATION STANDARDS}

A number of standards was defined during the last few years for very high data rate indoor communication at $60 \mathrm{GHz}$. The standards ECMA-387 and IEEE 802.15.3c were published in 2009 but no products were released so far based on them. The standard IEEE 802.11ad was published in December 2012, based on a standard that was previously developed by the WiGig Alliance [9]. This part of the IEEE 802.11 standard also recently got part of the Wi-Fi Alliance.

In the rest of this section we focus on IEEE 802.11ad as it is the most recent standard and due to it being part of a family of sub $6 \mathrm{GHz}$ transmission protocols. Nevertheless all three standards share some basic concepts, such as channel bandwidth, channelization and directional transmission with beamforming antennas.

\section{A. Overall topology}

The standard IEEE 802.11ad builds on top of the other IEEE 802.11 WPAN standards and introduces the extension to 60 GHz. In this extension one of the participating stations of a network or an access point (AP) takes the role of the PCP (personal basic service set control point) and provides basic timing information to all stations nearby. 
The radio transmissions are organized in so called beacon intervals, shown in Figure 3. Within a beacon interval there is a beacon header interval (BHI) and a data transfer interval (DTI). In the BHI one or multiple beacon signals (BTI) are transmitted by the PCP, some basic beamforming training (A$\mathrm{BFT}$ ) can be performed and announcements can be transmitted (ATI). The DTI consists of a contention-based access period (CBAP) and a contention free service period (SP).

While the stations can compete for access during the CBAP, the PCP schedules the transmissions according to the demand during the SP. Special care is taken to reduce interference that might occur through neighboring PBSS.

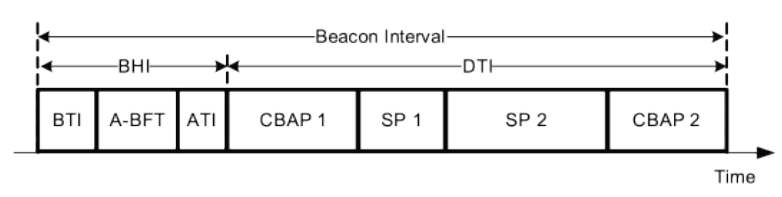

Figure 3. An IEEE 802.11ad beacon interval (BI) structure

\section{B. Beacon and discovery}

The beacon signal is transmitted by the PCP during the beacon transmission interval, as previously shown. During this interval the PCP can transmit multiple beacon signals in different spatial directions. As information on the spatial transmission is included in the beacon this is also the first step for the beamforming training.

\section{Beamforming support}

For any pair of communicating stations there is a total of 4 beamforming vectors that needs to be trained: transmit and receive vectors for each station. When the hardware supports reciprocity by using the same antenna array for transmission and reception and calibrated phase shifters, only one vector has to be trained per station.

The beamforming training is performed at the setup of a communication link and consists of the two phases sector-level sweep (SLS) and beam refinement protocol (BRP). A beam tracking protocol is used to update the beamforming vectors in an established link.

All protocols are based on the selection of the best performing beamforming vector out of a selection of candidates, for example from a code book. Multiple transmit beamforming vectors are applied sequentially and receiver feedback highlights the best one. This beamforming technique was developed for indoor environments and its applicability to outdoor environments with much larger distances and possibly higher time variance remains an open issue.

\section{Fast session transfer}

Fast session transfer (FST) allows to transfer a session from one physical channel to another channel (i.e. frequency band). A session is the state information that is kept in a pair of stations that have an established direct physical link. The FST allows the stations e.g. to switch to the $2.4 \mathrm{GHz}$ band when reaching the limits of coverage at $60 \mathrm{GHz}$. Each station can initialize or request the fast session transfer and it completes when both stations have established a connection in the new band.

From its design this approach is independent of the concrete implementation of the physical channels and does not rely on dedicated stable control channel. Such a channel however, as it is available in the split plane concept, could be used to improve the seamless transfer between the different channels or bands.

\section{E. Frame format}

The frame format is shown in Figure 4. A frame consists of a preamble, a header, the data payload and optional beamforming tracking (TRN) fields. The preamble is composed of a short training field (STF) and the channel estimation (CE) sequence. Both of these fields use special concatenations of Golay sequences. The short training field is used for AGC training, frequency offset estimation, synchronization and determines the type of frame (e.g. control frame, OFDM frame, single carrier frame). The channel estimation sequence is designed in a way that allows efficient calculation of the channel impulse response.

The header contains various fields, depending on the type of the frame. Fields can be the definition of the modulation and coding scheme (MCS) of the payload symbols, the number of payload bits, beamforming tracking requests, etc.

The optional beamforming tracking fields are inserted upon request and can be transmitted for receiver (R) or transmitter (T) tracking.

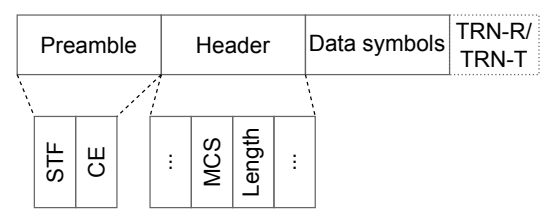

Figure 4. IEEE 802.11ad frame format

\section{F. Applicability to outdoor access}

The general topology of IEEE 802.11ad is similar to what would be needed for an evolution of wireless radio networks with millimeter-wave overlay cells. These small cells might advertise their existence with beacon signals and will coordinate the communication. They will also have to take care of beamforming training and tracking, which might become even more challenging in dynamic outdoor environments, compared to the indoor environments targeted by IEEE 802.11ad.

The fast session transfer is a building block that is also needed for outdoor access. Though its implementation in IEEE 802.11ad relies on a stable link in the old band. This might be problematic when this link gets suddenly lost. By relying on a separate, more stable control plane the reliability of the fast session transfer could be increased. 
The frame format and the physical layer (PHY) (not described here in detail) could form a basis for an millimeterwave outdoor access system. The challenges of initial synchronization, channel estimation, etc. are well addressed for wide bandwidth channels. Regular beam tracking is also included, though this might need to be extended.

\section{SPLit PLANE CONCEPT}

As introduced in section II the concept envisioned for the overlay of millimeter-wave small cells foresees a split of the control and data connection of the UE to the access network. Through this the UE can keep a main control-plane connection active, typically within a long-range macro cell, and activate user-plane connections to different base stations which provide the best data traffic bearers according to both user and network status. Different connection configurations can be possible in order to adapt to different network and traffic layouts [10]. User-plane connections can be established with both macro cells and small cells leaving room for the development of optimized resource allocation algorithms. In this scenario we can identify two types of mobility, a so-called small-scale mobility, where the UE moves within the coverage of a single control-plane macro cell and performs user-plane handovers through small cells, while traditional mobility occurs when the UE crosses macro cell boundaries.

\section{A. Time dynamic behavior}

Two aspects of time dynamic behavior that might have a great impact on the design of the split plane concept are worth mentioning. One is the behavior of a UE with respect to the coverage area of a small cell and the other are the implications of the kind of traffic that is requested by the UE.

1) UE behavior: Several possible behaviors of the UE with respect to its position within the macro cell can be identified: entering a small cell, staying or moving within a small cell, exiting a small cell. Even the type of mobility is important: very fast users with irregular trajectory can be difficult to serve with millimeter- wave small cells, while users with predictable trajectory can be served by properly allocating resources in advance.

2) Traffic model: The traffic model or type of traffic has a great impact on the way the control functionality should behave. While a traffic with fully buffered maximum demand simply requires a continuous high speed data connection two other cases are more challenging. One would be sporadic or regular high data traffic demand with gaps between transmissions (Fig. 5a) and the other would be low or medium rate continuous traffic (Fig. 5b).

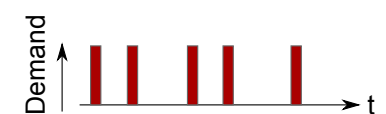

(a) sporadic

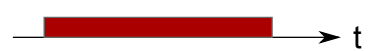

(b) constant
Figure 5. Traffic models
In the case of intermitted traffic the decision has to be made whether the connection between the UE and the millimeterwave small cell should remain active. When it is kept active the beamforming vectors have to be updated, even though the link is unused. In the case of low or medium rate traffic the decision has to be made whether the millimeter-wave or the legacy wireless connection should be used. For these decisions a number of factors, such as total traffic demand, link quality, power consumption of components or links have to be taken into account. Also the delay tolerance of the requested service is an important factor, it may or may not allow buffering techniques to help network reconfigurations.

\section{B. Logical localization of control functionality}

Despite UE double connectivity and control-/user-plane split, control functionalities should not be entirely relegated to one wireless interface. There are some control functions, like channel estimation and beamforming tracking, that must be carried out on the millimeter-wave link. Other functions, instead, can be partially or totally moved to the legacy $4 \mathrm{G}$ connection. Generally speaking, $4 \mathrm{G}$ connection is more avaialable, has higher reliability and requires fewer handovers than the millimeter connection, therefore, it can be used for exchanging information not directly related to the use of millimer-wave resources, but rather, to critical network management functions, like session setup, location update and context acquisition. In addition, control functionalities, having no strict bandwidth requirements, can waste millimeter-wave resources, which are better suited for high-rates and would be less efficiently utilized.

We can divide the control plane in two subplanes: macro cell control plane (on legacy 4G connection) and small cell control plane (on millimeter-wave link). We discuss about control function allocation on each subplane in the following paragraphs.

\begin{tabular}{c|c|c|c|c|c|c|}
\hline $\begin{array}{c}\text { Macro } \\
\text { cell }\end{array}$ & $\begin{array}{c}\text { cell } \\
\text { discovery }\end{array}$ & $\begin{array}{c}\text { context } \\
\text { acq. \& } \\
\text { man. }\end{array}$ & $\begin{array}{c}\text { session } \\
\text { setup }\end{array}$ & $\begin{array}{l}\text { location } \\
\text { update }\end{array}$ & & $\begin{array}{c}\text { resource } \\
\text { allocation }\end{array}$ \\
\hline $\begin{array}{c}\text { Small } \\
\text { cell }\end{array}$ & & & \multicolumn{1}{c|}{$\begin{array}{c}\text { beamform. } \\
\text { tracking }\end{array}$} & \\
\hline
\end{tabular}

Figure 6. Control functionality and possible localization on control plane

1) Cell discovery: The UEs discovery and attachment to a macro cell base station is made as usual. The discovery of millimeter-wave small cell base stations does not necessarily have to be performed continuously but only when needed or requested. Discovering available small cells becomes more complicated with the increased path loss in the millimeterwave bands. This requires either omnidirectional modes with low data rates and signal processing gain (e.g. spreading sequences) or directional antenna patterns with a certain gain. The support of a separated control-plane connection established with a macro cell becomes crucial to provide information for a fast discovery. The information can go from a minimal list of millimeter-wave small cell positions to a 
smart antenna configuration suggestion to connect to the best small cell.

2) Context acquisition and management: In order to make smart choices for cell discovery the system must rely on a rich context about UE and network status. The network collects information on UE position, antenna configurations, related channel quality and builds a context database that is used to predict the performance experienced by future UE requests at the same position. This information, together with UE type, capabilities, and traffic requirements, is conveyed to the resource allocation engine through the macro cell connection, which is more reliable.

3) Session setup: The session setup discussed here is the process of starting a communication between the UE and the access network on request by either entity. When the UE is in idle state it is connected to the legacy macro base station. It is assumed that the UE is not connected to a millimeter-wave link when idle, in order to reduce the energy consumption. This reduce both UE power consumption and network power consumption. Indeed, due to control-/user-plane separation, millimeter-wave base stations, which are typically deployed in large number and are mainly used to provide high-rate traffic bearers, can be switched off when no data session is active.

When the session is initiated by a request from the macro base station, the macro cellular control plane has to be used to alert the UE. Then the UE and the appropriate small cell have to initiate the directional millimeter-wave connection. This could be performed solely on the millimeter-wave control plane or with support on the macro cellular control plane. Such support context information could be for example position information of the UE, physical locations of small cells within the macro cell area, timing information, etc.

Once a millimeter-wave link is established, further control information can be transferred on either of both control planes.

4) Location update: As described above, the expected directionality of the millimeter-wave links requires a new kind of mobility support on the link or beam level. This is discussed as beamforming tracking. The traditional understanding of mobility, i.e. a UEs attachment to a certain macro base station or area, will undoubted remain on the legacy control plane, due to the much higher robustness of the link.

5) Beamforming tracking: The beamforming tracking is a crucial part of the millimeter-wave connectivity. As discussed in section III the directional millimeter-wave link continuously changes and updates of beamforming vectors have to be performed frequently. Such information is needed on both ends of the millimeter-wave link. This functionality will therefore undoubtedly be located at the small cell control plane.

6) Resource allocation: The resource allocation describes how and when the UE and the small cell should access the millimeter-wave channel. Depending on the granularity of the channel access and due to the increased complexity with directional communication this can generate a noteworthy amount of traffic. Taking IEEE 802.11ad as an example the small cell polls the UEs for their demand to communicate and allocates time slots. However, the use of macro cell con- trol plane allows to orchestrate the operation of surrounding millimeter-wave small cells. This, on one hand, facilitates the wireless medium access of millimeter-wave devices, on the other hand, allows to fine tuning available resources enable scenarios where UEs receive multiple streams from different millimeter-wave base stations and, vice versa, a millimeterwave base station simultaneously communicates with multiple UEs. In addition, interference coordination techniques can further improve the wireless resource exploitation. The following scenario can be envisioned: medium access and high-level connection management are carried out through macro cell control plane, while small cell control plane is involved in local millimeter-wave resource allocation and adaptation.

\section{CONCLUSIONS}

The introduction of the millimeter-wave frequency bands into future generations of mobile radio networks requires new architectural approaches and opens a new design space. In this paper we investigated the specific requirements of these high frequencies in the context of outdoor access systems. Based on a review of existing recent $60 \mathrm{GHz}$ indoor standards we investigated the design options for a split plane concept where data and control traffic can be transported on different physical interfaces (i.e. frequency bands).

While the localization of some functionality (e.g. location update, beamforming tracking) in such an architecture is very clear, the other functionality still offers a large design space. The exploration of the design space and the choice of a solution within this space relies on the knowledge of the whole system, from the physical propagation channel to the network architecture and type of traffic model. Further detailed investigations are necessary to fully leverage the potentials of the millimeter-wave spectrum in future mobile radio networks.

\section{ACKNOWLEDGMENT}

The research leading to these results has received funding from the European Union's Seventh Framework Program (FP7-ICT-2013-EU-Japan) under grant agreement number 608637 .

\section{REFERENCES}

[1] F. Boccardi, J. Heath, R.W., A. Lozano, T. Marzetta, and P. Popovski, "Five disruptive technology directions for 5G," Communications Magazine, IEEE, vol. 52, no. 2, pp. 74-80, February 2014.

[2] W. Roh, J.-Y. Seol, J. Park, B. Lee, J. Lee, Y. Kim, J. Cho, K. Cheun, and F. Aryanfar, "Millimeter-wave beamforming as an enabling technology for $5 \mathrm{G}$ cellular communications: theoretical feasibility and prototype results," Communications Magazine, IEEE, vol. 52, no. 2, pp. 106-113, February 2014.

[3] R. J. Weiler, M. Peter, W. Keusgen, E. Calvanese-Strinati, Antonio De Domenico, Ilario Filippini, Antonio Capone, Isabelle Siaud, Anne-Marie Ulmer-Moll, Alexander Maltsev, Thomas Haustein, and and Kei Sakaguchi, "Enabling 5G backhaul and access with millimeterwaves," in Conference on Networks and Communications (EuCNC), 2014 European, 2014

[4] E. Ben-Dor, T. S. Rappaport, Y. Qiao, and S. J. Lauffenburger, "Millimeter-wave $60 \mathrm{GHz}$ outdoor and vehicle AOA propagation measurements using a broadband channel sounder," in Global Telecommunications Conference (GLOBECOM 2011), 2011 IEEE. IEEE, 2011, pp. 1-6. 
[5] T. S. Rappaport, E. Ben-Dor, J. N. Murdock, and Y. Qiao, "38 GHz and $60 \mathrm{GHz}$ angle-dependent propagation for cellular \& peer-to-peer wireless communications," in Communications (ICC), 2012 IEEE International Conference on. IEEE, 2012, pp. 4568-4573.

[6] W. Keusgen, R. J. Weiler, M. Peter, and M. Wisotzki, "Propagation measurements and simulations for millimeter-wave mobile access in a busy urban environment," in 39th International Conference on Infrared, Millimeter, and Terahertz Waves, 2014.

[7] R. J. Weiler, M. Peter, W. Keusgen, and M. Wisotzki, "Measuring the busy urban $60 \mathrm{ghz}$ outdoor access radio channel," in Ultra-Wideband (ICUWB), 2014 IEEE International Conference on, 2014.

[8] A. Maltsev, A. Pudeyev, I. Bolotin, G. Morozov, I. Karls, M. Faerber, I. Siaud, A.-M. Ulmer-Moll, J.-M. Conrat, R. Weiler, and M. Peter, "MiWEBA Deliverable D5.1: Channel modeling and characterization," 2014. [Online]. Available: http://www.miweba.eu

[9] IEEE Standard for Information technology - Part 11: Wireless LAN Medium Access Control (MAC) and Physical Layer (PHY) Specification - Amendment 3: Enhancements for Very High Throughput in the $60 \mathrm{GHz}$ Band, IEEE Std., December 2012.

[10] T. Sakurai, S. Okasaka, T. Sotoyama, I. Siaud, A.-M. Ulmer-Moll, A. Capone, I. Filippini, A. D. Domenico, R. Weiler, and K. Sakaguchi, "MiWEBA Deliverable D1.2: Specification of architecture," 2014 [Online]. Available: http://www.miweba.eu 